\title{
Extension of Einstein's Law for Power-Law Fluid to Describe a Suspension of Spherical Particles: Application to Recycled Polymer Flow
}

\author{
Ghinwa El Hajj Sleiman, Isabelle Petit, Nadine Allanic, Sofiane Belhabib, Yannick Madec, Rémi Deterre (D) \\ Laboratoire GEPEA, UMR CNRS 6144, Université - IUT de Nantes (site de Carquefou), Nantes 44475, France
}

\begin{abstract}
In this work, Einstein's equation is extended considering a power-law suspending fluid without any Newtonian approximation. To validate the developed equation, an experimental setup is carried out. Polypropylene (PP) and polyethylene (PE) are injected at different volume fractions. The pressure drops measured in a cylindrical die are analyzed. The results show that the developed relationship allows better prediction of the viscosity of PP/PE blends compared to existing laws. During the recycling of $\mathrm{PP}$, some pollutants are likely to be present in the polymer, mostly PE which tends to form a heterogeneous melt with PP. At low volume fractions, PE disperses mostly as solid spheres in PP due to its higher viscosity, but the viscosity of the PP/PE mixtures is hard to predict. Several studies have derived equivalent viscosity equations for dispersed spherical suspensions in shearthinning polymers. Nevertheless, these equations mainly refer to Einstein's equation for suspended spheres in Newtonian fluids. POLYM. ENG. SCI., 59:E387-E396, 2019. @ 2019 Society of Plastics Engineers
\end{abstract}

\section{INTRODUCTION}

Heterogeneous systems consisting of particles suspended in a fluid medium make up a wide variety of materials of practical interest, both natural (slurries, debris flows, lavas, etc.) or manmade (concretes, food pastes, paints, etc.). This abundance explains why the behavior of these materials has been extensively studied from both, a theoretical and an experimental point of view $[1,2]$. However, obtaining a material with desired rheological characteristics, from components having known properties, is still challenging.

Given that filled polymers are very complex systems, they have been a research topic for the last decades. Often the filled polymers are processed in their molten state, thus the flow properties of the polymer-particle mixture are of great interest to the processing of the polymers and the final properties of the products [3, 4]. Despite the complexity of the filled polymers, mixtures of polymers of different viscosities are considered to be more complex. Most of the polymer blends are immiscible [5]. So, by mixing two polymers, complex interfaces are formed due to deformation, breakup and coalescence of droplets caused by shear and interfacial tension. For that reason, the morphological development of the polymer blends is difficult to predict [6-8] and their rheological properties can be written in function of the matrix fluid viscosity $\eta_{\mathrm{m}}$, the dispersed polymer

Correspondence to: R. Deterre; e-mail: remi.deterre@univ-nantes.fr Contract grant sponsor: Ministère de l'Enseignement Supérieur et de la Recherche.

DOI 10.1002/pen.25070

Published online in Wiley Online Library (wileyonlinelibrary.com).

(C) 2019 Society of Plastics Engineers viscosity $\eta_{\mathrm{d}}$, the volume fraction $\phi$ and the surface tension $\gamma[9,10]$. Of these factors, the ratio of the viscosities as well as the volume fraction will be of a great interest in the present work concerned by recycled polymers.

The increasing consumption of plastics inevitably leads to the production of large amount of plastic waste. But due to their unique properties, plastics can hardly be substituted by other materials, therefore, their collection and recycling is the only way, for the time being, to avoid environmental pollution. Knowing that Polypropylene (PP) and Polyethylene (PE) make up to $45 \%$ of the plastic production in the world [11], the study of the recycled matter behavior derived from these two polymers is therefore of great importance [12]. Combination of PP and PE is frequently found in polymer waste streams since they are used together in the manufacturing of many products like household items for instance but cannot be easily separated from each other [13]. Even though different methods of post-consumed polymer separation and sorting have been developed, the final product will always contain impurities and pollutants (up to $5 \%$ [14]) that will affect the mechanical and physical properties of the processed parts. As a consequence, we have studied in the present work the case of recycled PP, especially recycled PP polluted with PE. Most of the time, the recycled PP is made up of PP grade injection (low viscosity) containing a little amount of PE extrusion grade (high viscosity).

According to previous studies [15], at low volume fractions $(\phi<0.2)$, the ratio of the viscosity of the dispersed phase to that of the continuous phase greatly influence the morphology of different blends, where a droplet-dispersed phase structure occurs at a high viscosity ratio $\left(\kappa=\eta_{\mathrm{d}} / \eta_{\mathrm{m}}>>1\right)$. As the volume fraction $\phi$ increases, a tendency towards great irregularity is present with increasing shear rate. In this study, PP/PE mixtures are considered to behave as dilute blends where the PE polymer of higher viscosity is of low volume fraction $(\phi<0.2)$. Thus, PE is considered to be dispersed in PP matrix in the form of spherical droplets [16].

Einstein [17] was the first to study theoretically the suspension behavior in the dilute limit, where he derived-at low volume fractions $(\phi<0.03)$ - an analytical solution for the hydrodynamics around an isolated sphere which yields to Eq. 1:

$$
\eta^{\text {hom }}=(1+[\eta] \cdot \phi) \eta_{\mathrm{m}}
$$

where $\eta^{\text {hom }}$ is the homogenized viscosity of the suspension fluid and $[\eta]$ symbol is variously referred to as the "intrinsic viscosity" which takes the value $[\eta]=2.5$ for a Newtonian matrix fluid. The value of the coefficient $[\eta]$ has not been incontrovertibly validated, with different researchers favoring values covering the range $1.5 \lesssim[\eta] \lesssim 5[18]$.

For volume fractions up to $\phi \sim 0.1$, the Einstein equation was extended by Batchelor [19, 20] into Eq. 2: 


$$
\eta^{\text {hom }}=\left(1+2.5 \phi+6.2 \phi^{2}\right) \eta_{\mathrm{m}}
$$

In their Eqs. (1) and (2), both Einstein and Batchelor studied the viscosity of fluids containing solid spheres.

In 1932, Taylor [21] extended Einstein's work to liquids containing small drops of another liquid in suspension. In his work, Taylor made several assumptions:

1. The drops are so small that they remain nearly spherical.

2. There is no slipping at the surface of the drop.

The tangential stress parallel to the surface is continuous at the surface of the drop, so that any film which may exist between the two liquids merely transmits tangential stress from one fluid to another.

Thus, he replaced in Einstein (Eq. 1) the viscosity of a fluid containing solid spheres by a term containing the viscosities of the two fluids, considering by that the spheres as fluids, as shown in Eq. 3:

$$
\eta^{\text {hom }}=\left(1+2.5 \phi\left(\frac{\eta_{\mathrm{d}}+\frac{2}{5} \eta_{\mathrm{m}}}{\eta_{\mathrm{d}}+\eta_{\mathrm{m}}}\right)\right) \eta_{\mathrm{m}}
$$

As it is known, the above expressions were all carried out in the case of Newtonian fluid. However, polymers are in general viscoelastic and often show nonlinear (shear-thinning) behavior at high shear rates $[22,23]$, which is the case especially in injection process. In this case, the polymer is called pseudo-plastic [24] and its rheological behavior can be described by the power-law equation as in Eq. 4:

$$
\eta=K \dot{\gamma}^{n-1}
$$

where $n$ is the power-law index (smaller than unity) and $K$ is the consistency index (constant).

For that, researchers started to study the behavior of the suspensions in non-Newtonian fluids.

Chateau et al. [25] obtained, in a framework for nonlinear homogenization, the following linear-estimate for the equivalent viscosity of a dilute-suspension with a power-law matrix fluid (Eq. 5):

$$
\eta^{\text {hom }}=\left(1+\frac{7 n+3}{4} \phi\right) \eta_{\mathrm{m}}
$$

In 2012, Domurath et al. [26] proposed another approach for determining the equivalent viscosity in a nonlinear behavior of polymers. They named this approach the stress and strain amplification approach (SSAA; Eq. 6), where both the stress and the strain terms are amplified by different factors: $a_{s}$ and $a_{d}$ (Eq. 6)

$$
\eta^{\text {hom }}=\mathrm{a}_{\mathrm{s}} \cdot \mathrm{a}_{\mathrm{d}}^{2} \cdot \eta_{\mathrm{m}}\left(\mathrm{a}_{\mathrm{d}} \dot{\gamma}_{0}\right)
$$

The stress and the strain amplification factors are represented in Eqs. (7) and (8) respectively:

$$
\begin{gathered}
\mathrm{a}_{\mathrm{s}}=1+0.5 \phi \\
\mathrm{a}_{\mathrm{d}}=\frac{1}{1-\phi}
\end{gathered}
$$

As a continuation of their work, Domurath et al. [27] performed in 2015 a numerical study of a dilute suspension based on a Bird-Carreau model. An elongation flow of a Bird-Carreau fluid around a sphere was simulated and the effective viscosity of the dilute suspension for different applied rates of deformation and different power-law indices was obtained. Then, from the simulation results, they computed the intrinsic viscosity found in Eq. 9:

$$
[\eta]=2.5+\frac{47}{20}(n-1)+\frac{3}{10}(n-1)^{2}
$$

In this article, we have reviewed the most recent theories concerning rigid particles suspended in non-Newtonian fluids. However, the calculation of both Domurath [26] and Chateau [25] refer to Einstein's equation. In particular, Domurath uses Einstein equation for a Newtonian fluid (Eq. 1) in order to calculate his stress amplification factor. Concerning the equation of Chateau, he developed his relation (Eq. 5) for dilute suspensions, by using Einstein's relation in the case of Newtonian suspending fluid (Eq. 1). Concerning the most recent work of Domurath [27], although his work was numerical, he referred to Einstein equation in order to calculate some parameters.

For larger values of the volume fraction $\phi$, relations due to Mooney [28] (Eq. 10) and Krieger \& Dougherty [29] (Eq. 11) are often used for determining the viscosity $\eta^{\text {hom }}$, although these equations are empirical.

$$
\begin{gathered}
\eta^{\text {hom }}=\exp \left(\frac{\frac{5}{2} \phi}{1-\frac{\phi}{\phi_{\max }}}\right) \\
\eta^{\text {hom }}=\left(1-\frac{\phi}{\phi_{\max }}\right)^{-2.5 \phi_{\max }}
\end{gathered}
$$

where $\phi_{\max }$ is the volume fraction at maximum packing.

In this study, we recalculate the equation of Einstein considering the suspending fluid as a power-law fluid without any Newtonian approximation or numerical approach. We calculate the equivalent viscosity by equating the macroscopic energy with the microscopic one based on a previous study [30]. This approach applies to dilute suspensions of hard spherical particles where the interactions between particles can be neglected.

\section{EXTENSION OF EINSTEIN EQUATION FOR A POWER-LAW FLUID}

In order to understand the effect of the suspensions on the viscosity of a power-law behaving fluid, we have taken a similar approach to that studied in a previous paper for Newtonian fluid [30] and transposed it to the case of a power-law fluid. The application of this concept in the case of recycled polymers is the same as considering that PP as the medium fluid (matrix), while PE disperses as non-colloidal spherical rigid particles in PP.

Based on Einstein approach by Lauffer [30], we used the concept of an equivalent homogeneous fluid which exhibits the same flowing behavior as the polymer suspension. In his approach, Lauffer considers a representative elementary volume where he studies the rotation of the liquid without a change in the relative positions of the particles of the liquid, in addition to the viscous dissipation of the matrix liquid due to the displacement of the particles of the liquid. In our study, the viscosity of the equivalent fluid is defined as $\eta^{\text {hom }}$ and the shear rate of this flow is defined as $\dot{\gamma}_{0}$. 


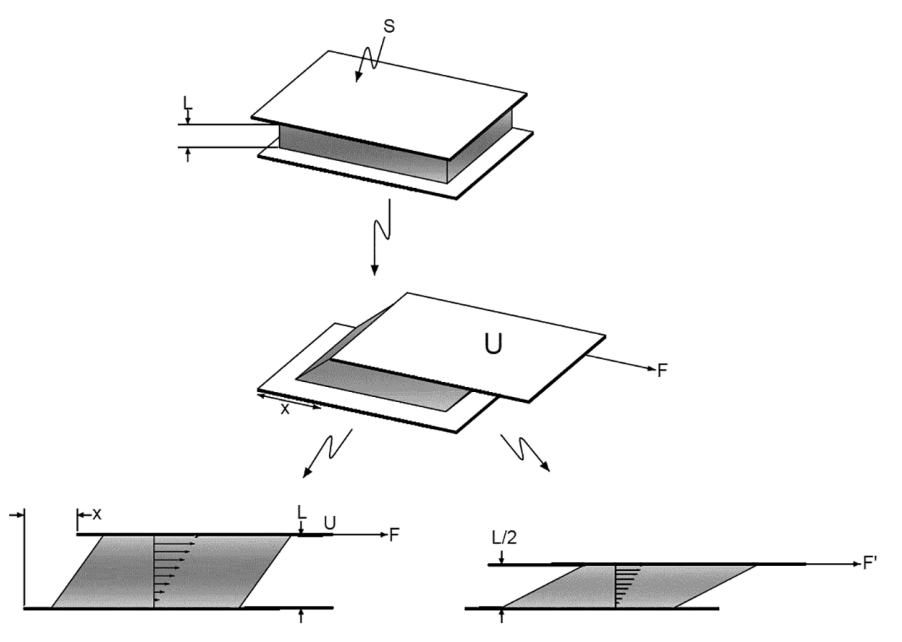

FIG. 1. Simple shear flow.

Then, by equating the macroscopic energy dissipated by the homogenized suspended fluid and the microscopic energy, we are able to conclude an equation relating the equivalent viscosity $\eta^{\text {hom }}$ with the volume fraction of the suspensions $\phi$, the power-law index $\mathrm{n}$ and the viscosity of the medium fluid $\eta_{\mathrm{m}}$.

\section{Viscosity of a Suspension of Spheres}

According to Domurath et al. [26] and Smallwood [31], the local tensor of the shear rate $\dot{\gamma}$, can be written as a function of the macroscopic shear rate $\dot{\gamma}_{0}$ as follows (Eq. 12):

$$
\dot{\gamma}=\frac{1}{1-\phi} \dot{\gamma}_{0}
$$

By replacing the suspended pseudo-plastic fluid by a fluid presenting an equivalent viscosity $\eta^{\text {hom }}$, then the total energy dissipated in this fluid is the sum of the energy due to the viscous dissipation of the matrix fluid $\left(W_{1}\right)$ plus the dissipated energy by the sphere rotation in the viscous fluid $\left(W_{2}\right.$; Eq. 13).

$$
W=W_{1}+W_{2}
$$

Considering a simple shear flow of a viscous fluid (viscosity $=\eta^{\text {hom }}$ ), the speed of the lower layer that is in contact with the fixed plate is considered to be zero while the upper layer that is in contact with the moving plate is considered to move with the speed U (Fig. 1).

Thus, the shear rate between the two plates is (Eq. 12):

$$
\dot{\gamma}=\frac{d u}{d y}
$$

The energy corresponding to the viscous dissipation is considered as the kinetic energy of the fluid in movement. This energy is determined multiplying the force $(F$; Fig. 1$)$ by the displacement of the fluid ( $x$; Fig. 1) [32] (Eq. 13).

$$
W_{1}=F \cdot x
$$

The force of a viscous fluid can be written as follow (Eq. 16):

$$
F=\eta \dot{\gamma} S
$$

Hence, by substituting Eq. 16 in Eq. 15, we get the expression for the energy $W_{1}$ (Eq. 17):

$$
W_{1}=F \cdot x=\eta \dot{\gamma} S \cdot x
$$

The energy of the viscous dissipation in a pseudoplastic matrix fluid $\left(W_{1}\right)$ is (Eq. 18):

$$
W_{1}=\eta_{\mathrm{m}} \dot{\gamma} S \cdot x=K \dot{\gamma}^{n-1} \dot{\gamma} S . x
$$

Where $S$ is the surface of the plate and $L$ is the distance between the two plates (Fig. 1).

As we have mentioned before, $x$ is the distance crossed by the moving plate, hence $x$ can be written as follow (Eq. 19):

$$
x=V . t=\dot{\gamma} L t
$$
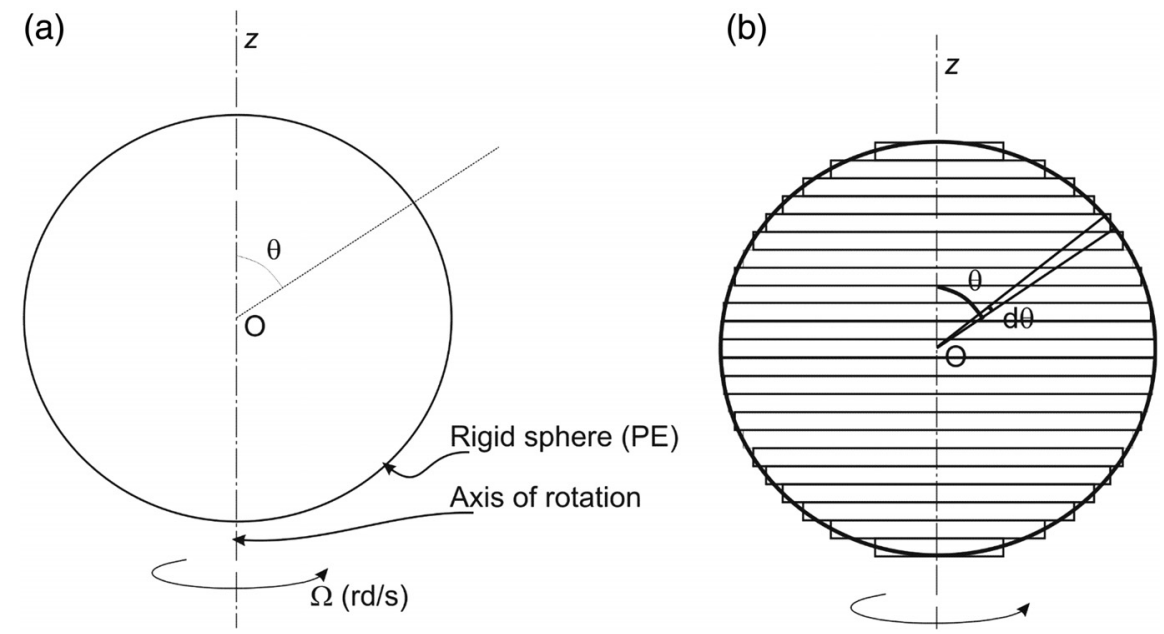

FIG. 2. (a) Sphere in rotation and (b) cross sectional diagram of a sphere rotating about a vertical axis divided into infinitesimal rings. 


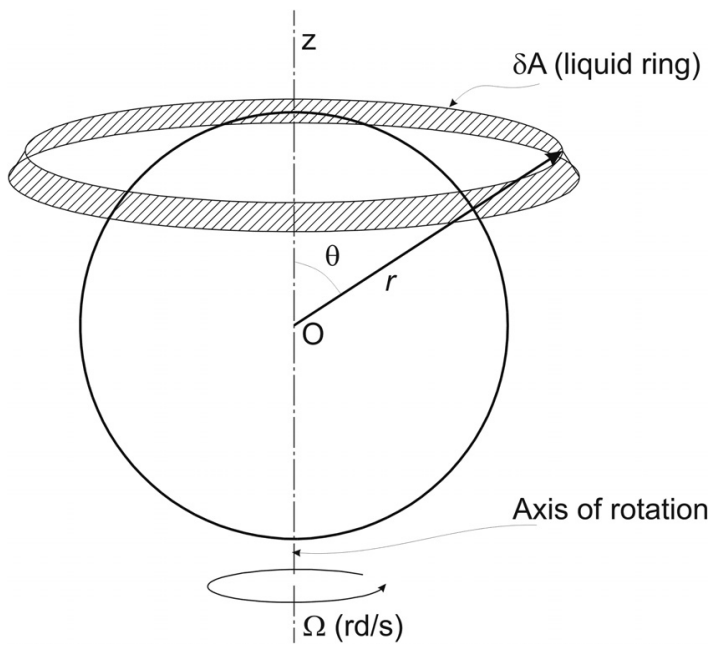

FIG. 3. Three-dimensional diagram of the sphere and the liquid rings.

After introducing the particles, the distance between the two plates corresponding to the fluid that can be deformed is reduced to $L(1-\phi)$. Hence, Eq. 18 can be written as follow (Eq. 20):

$$
W_{1}=K \dot{\gamma}^{n-1} \dot{\gamma} S . \dot{\gamma} L(1-\phi) t
$$

By replacing the shear rate term from Eq. 12 in Eq. 20, we get (Eq. 21):

$$
W_{1}=K \frac{\dot{\gamma}_{0}^{n+1}}{(1-\phi)^{n}} \mathrm{SL} t
$$

\section{Energy Dissipated by the Sphere Rotation}

In a cylindrical coordinate system, we consider a sphere of radius $r_{l}$ (representing the PE droplet), rotating about the vertical axis (z) with an angular velocity $\Omega(r d / s)$ (Fig. 2a).

The surface of the sphere can be divided into a very large number of rings of radius (Fig. 2b), $r_{1} \cdot \sin \theta$, and of small finite thickness, $r_{l} . \delta \theta$, rotating about the vertical axis $(\mathrm{z})$. At a distance $r$ from the center of the sphere for a particular value of $\theta$, a liquid ring (representing PP) of radius $r \cdot \sin \theta$ and thickness $r . \delta \theta$ is considered (Fig. 3).

Thus, the area of the shearing surface, $\delta A$ (Fig. 4), of this liquid ring is (Eq. 22):

$$
\delta A=2 \cdot \pi \cdot r \cdot \sin \theta \cdot r \cdot \delta \theta=2 \cdot \pi \cdot r^{2} \cdot \sin \theta \cdot \delta \theta
$$

To calculate the shear rate (Eq. 23), we consider the flow of fluid in a Couette geometry (Fig. 4a), where the surface of the sphere is considered as the rotating inner cylinder with a rotational speed of $\Omega$, while the distant liquid $(r \rightarrow \infty)$, which describes a dilute solution, is considered as the stationary outer cylinder (Fig. 4b).

In a cylindrical coordinate system, the shear rate is as follows [33] (Eq. 23):

$$
\dot{\gamma}(r)=\frac{v(r)}{r}-\frac{d v(r)}{d r}
$$

where $v(r)$ is the tangential velocity of the liquid ring.

Considering a Couette flow (Fig. 5), the tangential velocity $v(r)$ can be calculated as follows [33] (Eq. 24):

$$
v(r)=\frac{R_{1}^{\frac{2}{n}} \Omega}{R_{2}^{\frac{2}{n}}-R_{1}^{\frac{2}{n}}}\left[\frac{R_{2}^{\frac{2}{n}}-r^{\frac{2}{n}}}{r^{\frac{2-n}{n}}}\right]
$$

If we consider the infinitesimal surface $\delta A$ from Fig. 3 as a Couette flow with the coordinates presented in Fig. $4 \mathrm{~b}$, then the parameters of Eq. 24 must be replaced as follows: $R_{1}$ is replaced by $r_{1} \sin \theta, r$ is replaced by $r \sin \theta$ and $R_{2}$ is replaced by $r_{2} \sin \theta$, which yields to Eq. 25.

$$
v(r)=\frac{r_{1} \sin \theta^{\frac{2}{n}} \Omega}{r_{2} \sin \theta^{\frac{2}{n}}-r_{1} \sin \theta^{\frac{2}{n}}}\left[\frac{r_{2} \sin \theta^{\frac{2}{n}}-r \sin \theta^{\frac{2}{n}}}{r \sin \theta^{\frac{2-n}{n}}}\right]
$$

In the case of dilute little spherical particles, $r_{2}$ is considered as infinite [17]. This leads to the following simplified form of $v$ (r) (Eq. 26):

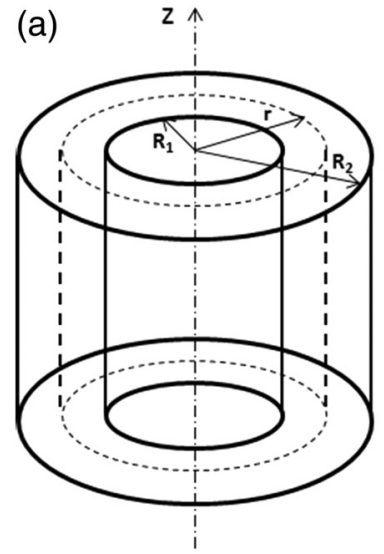

Axis of rotation (b)

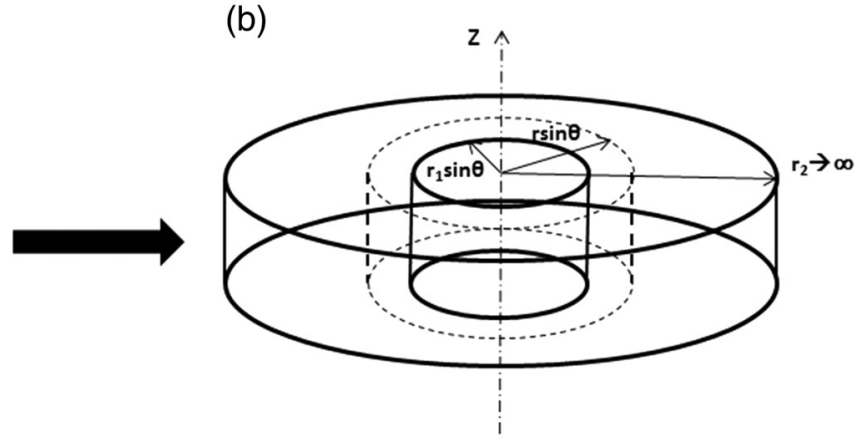

Axis of rotation

FIG. 4. (a) Flow in a classical Couette and (b) flow in a Couette where spherical rings are considered cylinders. 


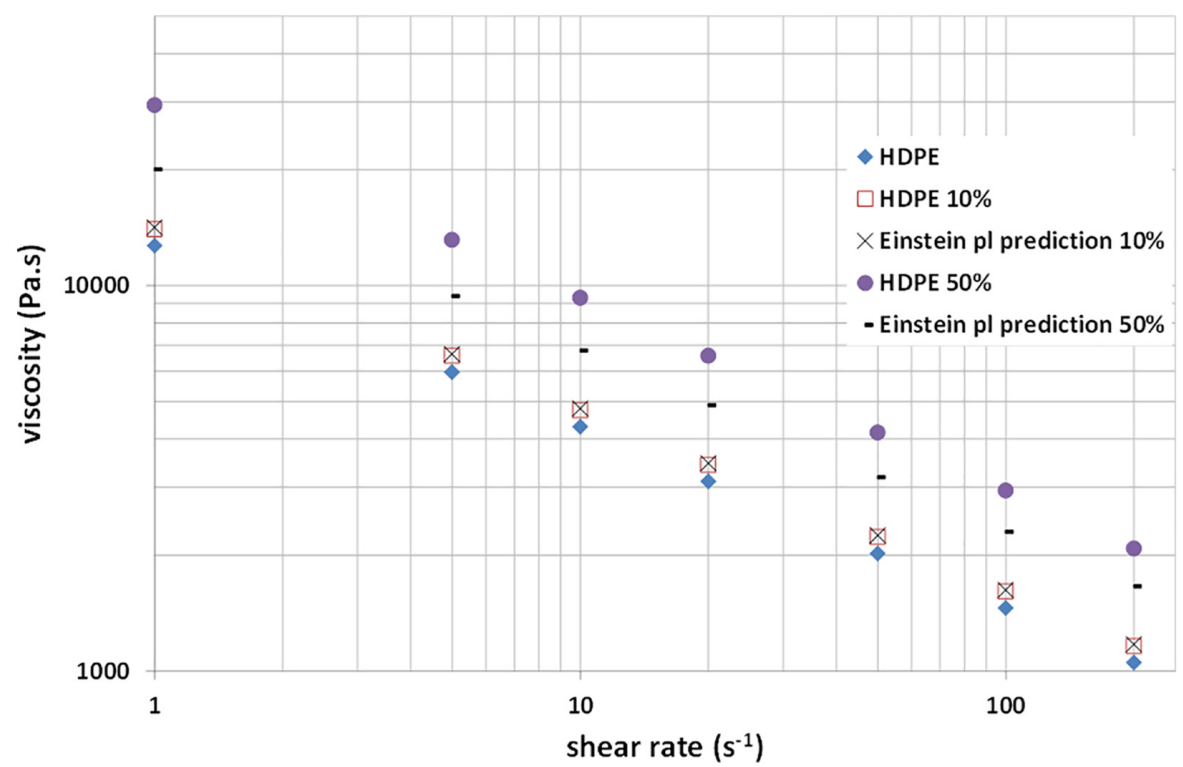

FIG. 5. The viscosities of pure high density polyethylene (HDPE), HDPE with fibers 10\% weight fraction, HDPE with fibers $50 \%$ weight fraction compared with the viscosities predicted by the Einstein power law model.

$$
v(r)=\frac{r_{1^{\frac{2}{n}}} \sin \theta \Omega}{r^{\frac{2-n}{n}}}
$$

According to Eq. 23, the tangential velocity expression (Eq. 26) allows us to calculate the shear rate $\dot{\gamma}(r)$ (Eq. 27):

$$
\dot{\gamma}(r)=\frac{r_{1^{\frac{2}{n}}} \sin \theta}{r^{\frac{2}{n}}} \Omega\left(\frac{2}{n}\right)
$$

The shear rate in the fluid at the surface of the sphere is $\dot{\gamma}\left(r=r_{1}\right)$ and calculated in Eq. 28:

$$
\dot{\gamma}=\Omega\left(\frac{2}{n}\right) \sin \theta
$$

The infinitesimal tangential friction force $\delta F$ exerted at the surface of the ring at the surface of the sphere $(\delta A$ at the position $\left.r=r_{1}\right)$ is the mathematical product of the viscosity by the surface and by the shear rate (Eq. 29):

$$
\delta F=2 \pi K r_{1}{ }^{2}\left[\Omega\left(\frac{2}{n}\right) \sin \theta\right]^{n} \sin \theta \delta \theta
$$

The infinitesimal torque $\delta M$ exerted at the surface of the ring from the sphere, is the mathematical product of the force by the distance to the $z$ axis (Eq. 30):

$$
\delta M=\delta F r_{1} \sin \theta=2 \pi K r_{1}{ }^{3} \Omega^{n}\left(\frac{2}{n}\right)^{n} \sin \theta^{n+2} \delta \theta
$$

The energy concerning the volume of the spherical particles, exerted during a rotation is the mathematical product of the torque $\delta M$ by the rotation speed $\Omega$ per the sphere volume in the same time interval $t$ (Eq. 31):

$$
\delta W_{2}=\frac{3}{2}\left(\frac{2}{n}\right)^{n} K \Omega^{n+1} \sin \theta^{n+2} \delta \theta \mathrm{t}
$$

By replacing the rotation speed $\Omega$ by the relationship versus the shear rate (Eq. 28), we get the expression of the energy exerted on a sphere annulus (Eq. 32):

$$
\delta W_{2}=\frac{3}{2}\left(\frac{n}{2}\right) K \dot{\gamma}^{n+1} \sin \theta \delta \theta \mathrm{t}
$$

In order to get the energy dissipated on the whole sphere, we integrate the local energy $\delta \mathrm{W}$ for $\theta$ varying from 0 to $\pi$, (Eq. 33):

$$
\Delta W_{2}=\frac{3}{2} n K \dot{\gamma}^{n+1} t
$$

Considering that there are $m$ spheres of radius $r_{1}$ in the fluid and by introducing the value of the volume fraction $\phi$, the whole energy dissipated by the $m$ spheres can be written as follows (Eq. 34):

$$
W_{2}=m \frac{\mathrm{V}_{\text {spheres }}}{V_{t}} \Delta \dot{W}_{2}=\phi \Delta \dot{W}_{2}=\frac{3}{2} n \phi K \dot{\gamma}^{n+1} t
$$

If we replace $\dot{\gamma}$ by its expression depending on $\dot{\gamma}_{0}$ according to Eq. 21, we get Eq. 35:

TABLE 1. Physical and rheological properties of PP and PE.

\begin{tabular}{cccccc}
\hline & $\begin{array}{c}C_{p} \\
\mathrm{~J} \cdot \mathrm{Kg}^{-1} \cdot \mathrm{K}^{-1}\end{array}$ & $\begin{array}{c}\rho \\
\mathrm{Kg} \cdot \mathrm{m}^{-3}\end{array}$ & $\begin{array}{c}\lambda \\
\mathrm{W} \cdot \mathrm{m}^{-1} \cdot \mathrm{K}^{-1}\end{array}$ & $\mathrm{n}$ & $K$ \\
\hline PPC 9642 & 2,720 & 905 & 0.17 & 0.332 & 4,636 \\
PE 2042E & 2,360 & 795 & 0.30 & 0.258 & 47,707 \\
\hline
\end{tabular}

$\mathrm{PE}$, polyethylene; PP, polypropylene. 


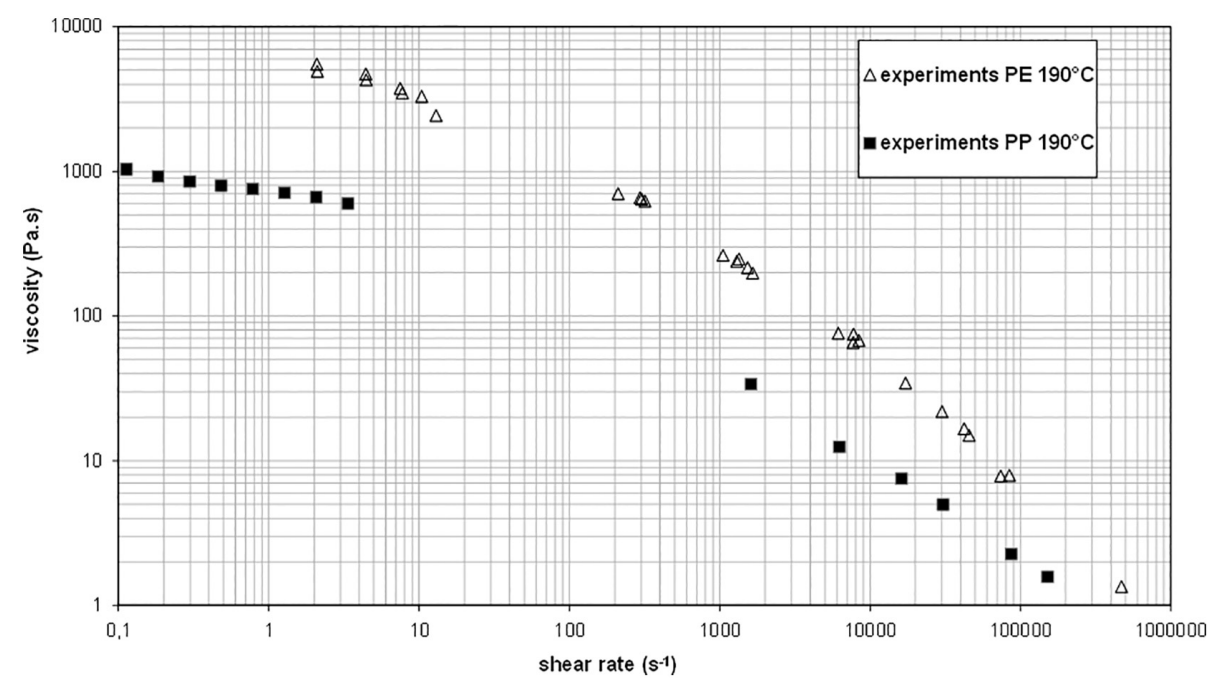

FIG. 6. The viscosities of PP and PE as a function of the shear rate. PE, polyethylene; PP, polypropylene.

$$
W_{2}=\frac{3}{2} n \phi K \frac{\dot{\gamma}_{0}^{n+1}}{(1-\phi)^{n+1}} V_{t} t
$$

As said in Eq. 11, the total energy $W$ is equal to the sum of the $W_{1}$ and $W_{2}$, this leads to (Eq. 36):

$$
\begin{gathered}
W=\mathrm{W}_{1}+W_{2}=K \frac{\dot{\gamma}_{0}^{n+1}}{(1-\phi)^{n}} \mathrm{SLt}+\frac{3}{2} n \phi K \frac{\dot{\gamma}_{0}{ }^{n+1}}{(1-\phi)^{n+1}} V_{t} t \\
\eta_{\mathrm{hom}} \dot{\gamma}_{0}^{2}=K \dot{\gamma}_{0}^{n+1}\left[\frac{1}{(1-\phi)^{n}}+\frac{3}{2} n \frac{\phi}{(1-\phi)^{n+1}}\right]
\end{gathered}
$$

Considering Eq. 30, we can identify the equivalent viscosity as follows (Eq. 37):

$$
\eta^{\text {hom }}=\eta_{\mathrm{m}} \frac{1}{(1-\phi)^{n}}\left[1+\frac{3}{2} n \frac{\phi}{(1-\phi)}\right]
$$

Where $\eta_{\mathrm{m}}$ is the viscosity of the fluid matrix and is represented by the power-law relation: $\eta_{\mathrm{m}}=K \dot{\gamma}_{0}^{n-1}$.
Considering that $\phi \ll 1$, we can use the limited development of $(1-\phi)$, which leads to Eq. 38 :

$$
\eta^{\text {hom }}=\eta_{\mathrm{m}}(1+n \phi)\left[1+\frac{3}{2} n \phi(1-\phi)\right]
$$

Considering that $\phi \ll 1$ leads also to neglect the terms depending on $\phi^{i}$ with $i \geq 2$, then Eq. 38 reduces to Eq. 39:

$$
\eta^{\text {hom }}=\eta_{\mathrm{m}}(1+2.5 n \phi)
$$

This relation was also observed experimentally by antecedent studies $[34,35]$ where they indicated that the intrinsic viscosity is approximately $\frac{5}{2} \mathrm{n}$.

We remark that if $n=1$, then Eq. 39 becomes equal to Einstein's equation (Eq. 40):

$$
\eta^{\text {hom }}=\eta_{m}(1+2.5 \phi)
$$

Polychronopoulos et al. [36] have studied the viscosity of heavily filled high density polyethylene (HDPE) with natural fibers. In their work, they have measured the viscosity of

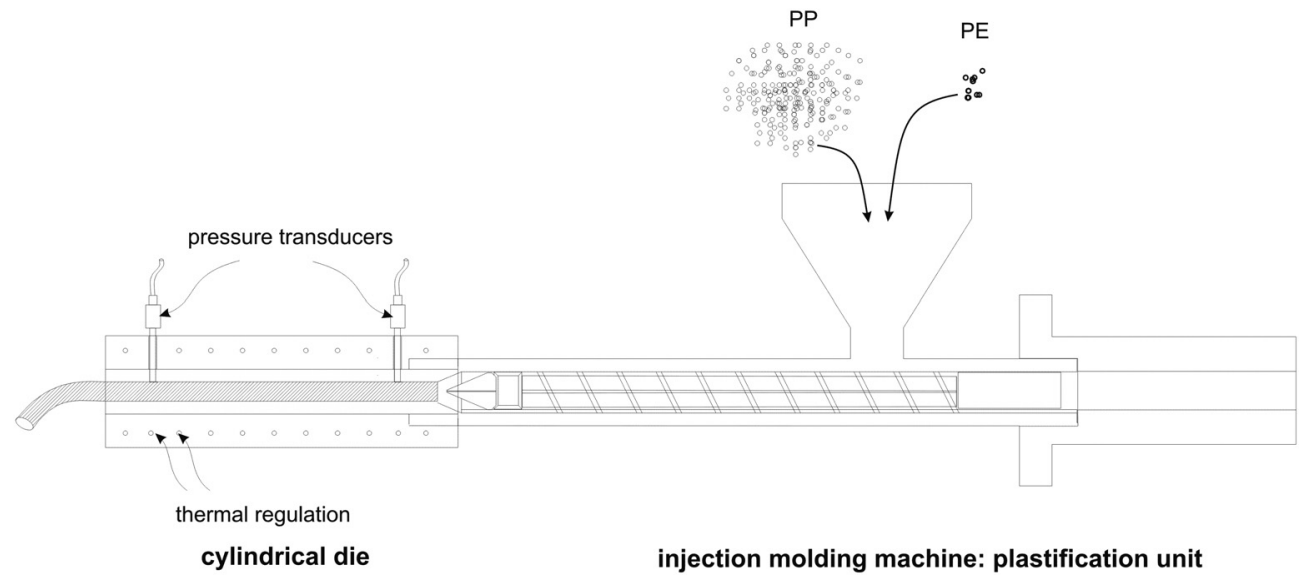

FIG. 7. Instrumented die for measuring the pressure drop. 


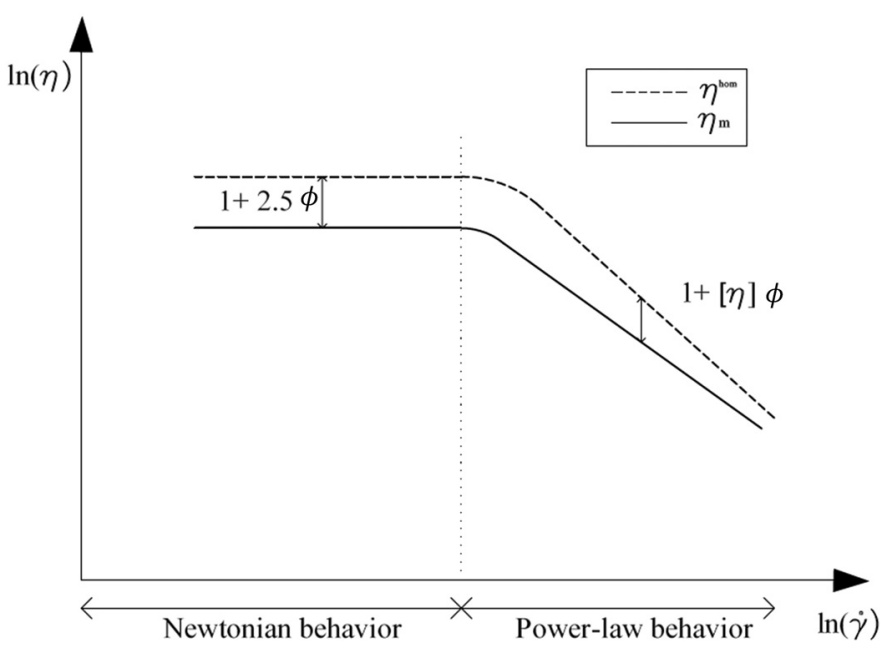

FIG. 8. Amplification of the viscosity in a suspension fluid.

filled HDPE at different weight fractions. So, in order to validate our equation, we have compared the viscosities predicted with our model, with the viscosities measured in the Polychronopoulos et al.'s work. The results are presented in Fig. 5.

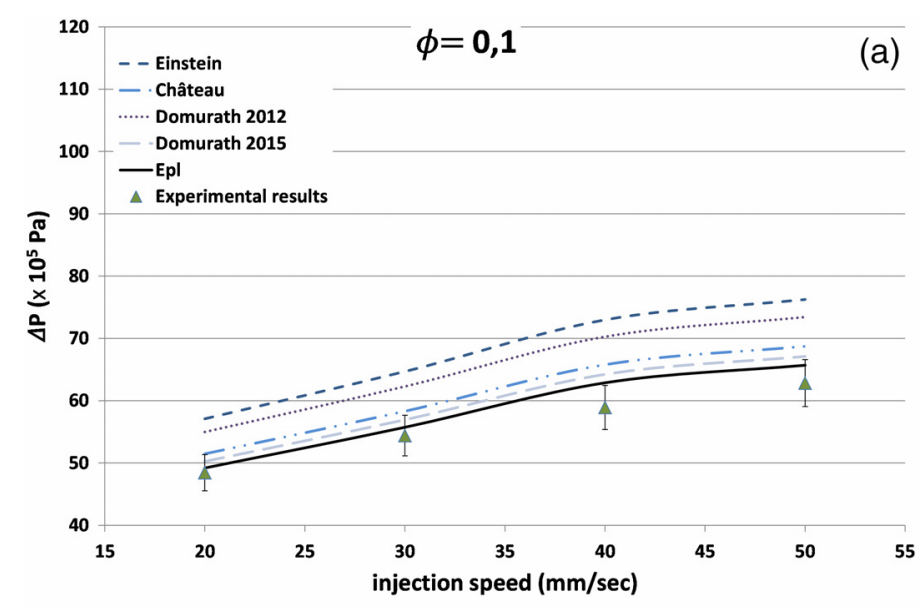

\section{)}

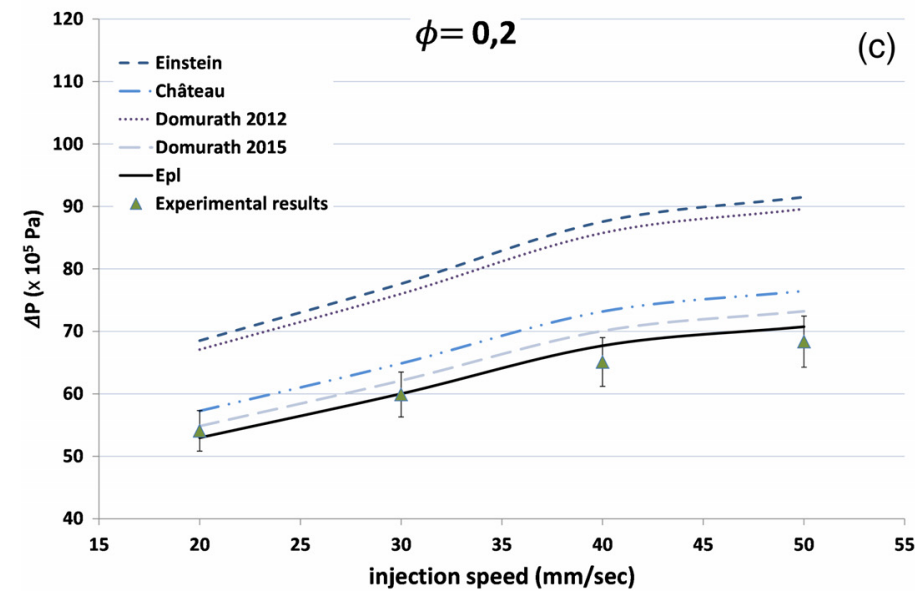

The results in Fig. 5 show that the viscosities predicted by the equation developed in this work are in good agreement with the viscosities measured in the study of Polychronopoulos et al. for volume fractions $<10 \%$. As the volume fraction increases, the Epl model departs from the experimental results which can be explained by the fact that as the volume fraction increases, the particle-particle interactions as well as the differences in the morphological development can no longer be neglected.

\section{EXPERIMENTAL SECTION}

Despite the validation of the model with experimental results found in literature, we have decided to validate the improvement brought by the Epl "Einstein power-law" (Eq. 39) presented in the section "Extension Of Einstein Equation For A Power-Law Fluid", with our own experimental results. The used experimental setup is described below.

\section{Materials}

The materials used in this work are PPC 9642 Total Petrochemicals and PE DOWLEX 2042E (Table 1). The density $(\rho)$ of the material is measured as the ratio on the mass flow rate and the volume flow rate by the melt flow index (MFI) apparatus at $190^{\circ} \mathrm{C}$, the specific heat $\left(C_{p}\right)$ is measured by the differential
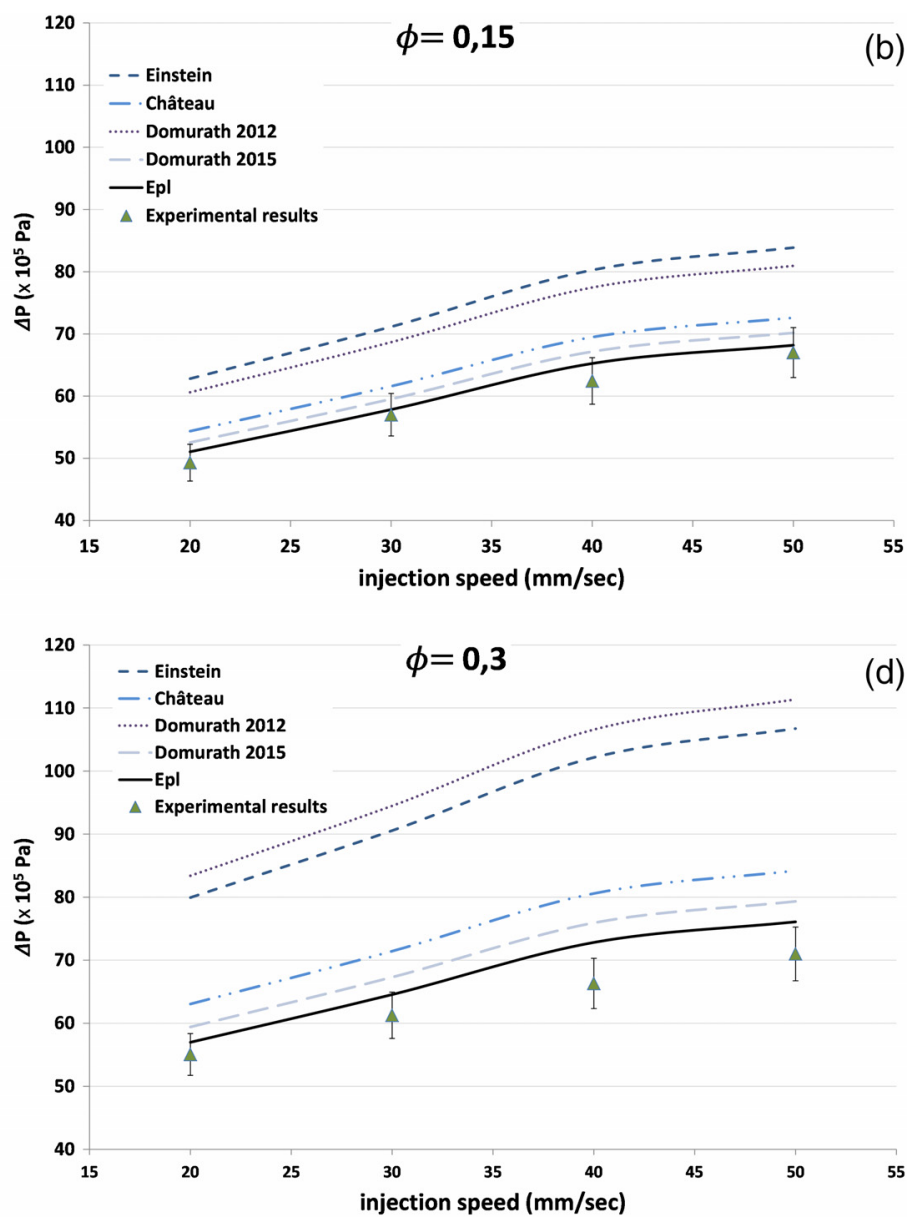

FIG. 9. The pressure drop in the die at different injection speeds compared to the mixing laws Epl, Chateau, Domurath, and Einstein (a: $\phi=0.1, \mathrm{~b}: \phi=0.15, \mathrm{c}: \phi=0.2$, and $\mathrm{d}: \phi=0.3$ ). 


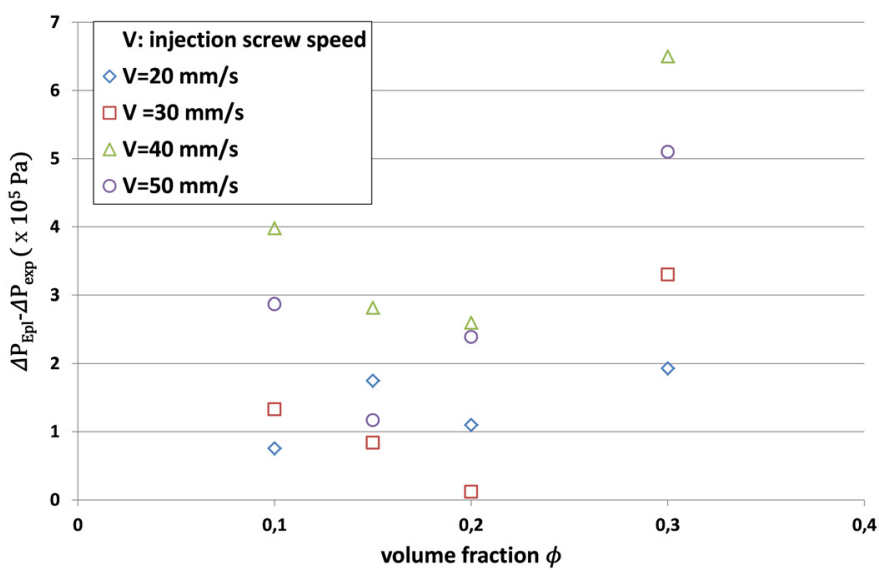

FIG. 10. Variation of the difference between Epl model and experimental results as a function of the volume fraction at different injection speeds.

scanning calorimetry (DSC) [37] while the value of the thermal conductivity $(\lambda)$ is taken from literature. [38]

On the other, we have measured the viscosities of PP and PE with a rheometer that was developed in a previous study [39]. The rheological behavior of the different polymers is presented in Fig. 6.

At low shear rates, the viscosities of each polymer were measured with a rheometer plan. In Fig. 6, we have showed the evolution of the viscosity as a function of the shear rate for PP and PE at $190^{\circ} \mathrm{C}$.

Table 1 represents the physical and rheological properties of the polymers PP and PE used in this study.

\section{Experimental Setup}

The aim of the experimental setup is to allow the measurement of the pressure drop, which is tightly related to the viscosity, of the injected PE/PP mixtures. A die that was developed in our laboratory in a previous work [40] (Fig. 7) is used in this study.

The device is a cylindrical die of $300 \mathrm{~mm}$ length with outer and inner diameters of 50 and $10 \mathrm{~mm}$ respectively. Hot wires of $2 \mathrm{~mm}$ diameter are used to regulate the temperature of the device. The heating system is divided into five zones allowing effective temperature control. The die is equipped with two pressure sensors of type KISTLER 6159A. The first is located at the inlet while the second is located at the outlet, so as to measure the pressure drop during the test. In his work, Launay [40] developed this setup with two temperature measuring cells (TMC) implemented in the die. These TMCs, that allow the measurement of the temperature in the flow, are not used in this work given their intrusive nature. We use the designed device (Fig. 7) as a pressure measuring device. The die is mounted at the exit of a process machine-in our case an injection molding (Milacron Elektron 50).

\section{RESULTS AND DISCUSSION}

The flow tests are performed by varying the injection speed $\mathrm{V}$ from 20 to $50 \mathrm{~mm} / \mathrm{s}$. During the experiments, the injection molding and runner temperatures are controlled in order to get the most homogeneous temperature of the polymer melt around $190^{\circ} \mathrm{C}$. The different laws introduced in section 1 are used to predict the pressure drop and then compared to experiment. The effect of adding rigid spheres on the viscosity is studied at a constant shear rate [26] (Fig. 8) where $[\eta]$ is the intrinsic viscosity.

So, by substituting $\eta$ by $\tau / \dot{\gamma}$, where the shear stress is given by Eq. 41 :

$$
\tau=\frac{\Delta P \cdot R}{2 L}
$$

We get the relation in terms of pressure drop (Eq. 42):

$$
\Delta P^{\text {hom }}=(1+2.5 n \phi) \Delta P_{m}
$$

Figure $9 \mathrm{a}-\mathrm{d}$ shows the pressure drop predicted by the different mixing laws-discussed in section "Introduction"-compared to the measured one in the die at different injection speeds for various volume fractions $\phi=0.1,0.15,0.2$, and 0.3 .

Whatever the different volume fractions and the different injection speeds are, Einstein Newtonian model is the worst to predict the pressure drop. Despite, the improvement brought by Domurath 2012 model and Chateau model, the predictions remain quite unsatisfactory. However, Domurath 2015 and Epl models give the closest prediction with respect to the measured pressure drop with a better prediction brought by the latter developed in this work.

In Fig. 10, we plot the difference between the predicted pressure drops by Epl model and the experimental results. As we can see, the difference increases as the volume fraction increases. This discrepancy is due to the fact that the conditions taken into consideration-namely small volume fraction and spherical rigid particles-in developing the Epl model no longer exist and new phenomenon such as particles interaction and coalescence might be taking place [41]. The difference also increases by increasing the injection speed hence increasing the shear rate. Indeed, this behavior can be explained by the fact that as the shear rate increases the dispersed particles will stretch, thus losing their spherical shape.

\section{CONCLUSION}

In the present article, we established a relationship in order to describe the equivalent viscosity in the case of suspended rigid spheres in a power-law behaving fluid. Unlike the reported models in literature, our calculation is based on the principle used by Einstein in his model but without any Newtonian approximation. The obtained equation predicts the equivalent viscosity taking into consideration the power-law index and the volume fraction.

The physics used here does not include any effects related to particles interaction, visco-elasticity, particle deformation or any other physicochemical effect. For that reason, its scope is limited to the cases where the interaction between the particles and the matrix fluid is purely hydrodynamic.

Due to the high viscosity ratio between PE and PP $(\kappa>>1)$, $\mathrm{PE}$ is assumed to behave as dispersed rigid particles in PP. We have shown that the equation developed in this work is in good agreement, for concentrations up to $\phi \sim 0.2$, with the experimental results and predicts the pressure drop better than the models found in literature which the authors are aware of. Moreover, the intrinsic viscosity of the developed model in this work $([\eta]=2.5 \mathrm{n})$ matches also the intrinsic viscosity found experimentally by other authors [42].

However, the developed equation Epl has limits when it comes to fluid mixtures of similar viscosities where the morphology of 
the blend changes as well as at high volume fractions where the interaction forces between the particles are no longer negligible.

For future work, improvement of this equation can be made by taking into consideration the viscosity of the dispersed particles and the deformation of the particles dispersed as well.

\section{ACKNOWLEDGMENTS}

This research was funded by the Ministère de l'Enseignement Supérieur et de la Recherche. We are also grateful to Total Petrochemicals for the material supply.

\section{ABBREVIATIONS}

DSC differential scanning calorimetry

Epl Einstein power-law

MFI melt flow index

PE polyethylene

PP polypropylene

SSAA stress and strain amplification approach

\section{NOTATION}

$\gamma \quad$ surface tension $(\mathrm{N} / \mathrm{m})$

$\dot{\gamma}$ macroscopic shear rate $\left(\mathrm{s}^{-1}\right)$

$\dot{\gamma}_{0} \quad$ applied rate-of-strain $\left(\mathrm{s}^{-1}\right)$

$\eta^{\text {hom }}$ equivalent viscosity (Pa.s)

$\eta_{\mathrm{d}} \quad$ viscosity of the dispersed polymer (Pa.s)

$\eta_{\mathrm{m}} \quad$ viscosity of the matrix polymer (Pa.s)

$[\eta] \quad$ intrinsic viscosity (reverse concentration)

$\theta$ angle between a sphere ring and the axis of rotation (rd)

$\kappa \quad$ viscosity ratio (1)

$v \quad$ tangential speed in Couette $(\mathrm{m} / \mathrm{s})$

$\phi \quad$ volume fraction of the dispersed polymer

$\Omega$ rotational speed at the surface of the rigid sphere $(\mathrm{rd} / \mathrm{s})$

\section{Symbols}

A shearing area $\left(\mathrm{m}^{2}\right)$

$\mathrm{a}_{\mathrm{s}}$ stress amplification factor

$\mathrm{a}_{\mathrm{d}}$ strain amplification factor

$F$ tangential frictional force $(\mathrm{N})$

$K$ consistency index $\left(\mathrm{Pa}_{\mathrm{s}} \mathrm{s}^{\mathrm{n}+1}\right)$

$M$ Torque on the surface of the rigid sphere (N.m)

$m$ number of the suspended spheres

$n$ power-law index

$\Delta P$ pressure drop $(\mathrm{Pa})$

$R$ radius of the flow channel $(\mathrm{m})$

$r$ radius of the liquid sphere $(\mathrm{m})$

$r_{1}$ radius of the rigid sphere $(\mathrm{m})$

$W$ total energy dissipated in the equivalent homogeneous fluid (J)

$W_{1}$ viscous dissipation of the fluid matrix (J)

$W_{2}$ energy dissipated by the flow of the fluid around $\mathrm{m}$ spheres (J)

\section{REFERENCES}

1. A.d.S. Sirqueira, D. Teodoro Júnior, M.d.S. Coutinho, S. Neto, A.S. Da, A.d.A. Silva, B.G. Soares, A.d.S. Sirqueira, D. T. Júnior, M.d.S. Coutinho, S. Neto, A.S. Da, A.d.A. Silva, and B.G. Soares, Polímeros, 26(3), 215 (2016).

2. D.S. Bolintineanu, G.S. Grest, J.B. Lechman, F. Pierce, S.J. Plimpton, and P.R. Schunk, Comput. Part. Mech., 1(3), 321 (2014).

3. G. Schmidt and M.M. Malwitz, Curr. Opin. Colloid Interface Sci., 8(1), 103 (2003).
4. Y.P. Mamunya, V.V. Davydenko, P. Pissis, and E.V. Lebedev, Eur. Polym. J., 38(9), 1887 (2002).

5. C.W. Macosko, Macromol. Symp., 149(1), 171 (2000).

6. M. Minale, P. Moldenaers, and J. Mewis, Macromolecules, 30 (18), 5470 (1997).

7. B.D. Favis and D. Therrien, Polymer, 32(8), 1474 (Jan. 1991).

8. R. Gonzalez-Nunez, B.D. Favis, P.J. Carreau, and C. Lavallée, Polym. Eng. Sci., 33(13), 851 (1993).

9. M. Vonka and M. Soos, AICHE J., 61(8), 2403 (Aug. 2015).

10. C. Carrot, S. Mbarek, M. Jaziri, Y. Chalamet, C. Raveyre, and F. Prochazka, Macromol. Mater. Eng., 292(6), 693 (2007).

11. PlasticsEurope - Plastiques 2010 : faits et chiffres. 2009. Available at: https://www.plasticseurope.org/fr/resources/publication/ 280-plastiques-2010-faits-et-chiffres

12. N. Equiza, W. Yave, R. Quijada, and M. Yazdani-Pedram, Macromol. Mater. Eng., 292(9), 1001 (2007).

13. S. Bertin and J.-J. Robin, Eur. Polym. J., 38(11), 2255 (2002).

14. "PP and PE separation - Make more money from plastics," Impact Sol., 2015. Available at: https://www.impact-solutions.co. uk/pp-and-pe-separation-equipment/

15. H.M. Lee and O.O. Park, J. Rheol., 38(5), 1405 (1994).

16. K. Askar, "Polymer blend de-mixing and morphology development during tube flow," University of Toronto, Toronto, 1999.

17. A. Einstein, Annal. Phys., 324, 289 (1906).

18. S. Mueller, E.W. Llewellin, and H.M. Mader, Proc. R. Soc. Lond. Math. Phys. Eng. Sci., 466(2116), 1201 (2010).

19. G.K. Batchelor, J. Fluid Mech., 83(1), 97 (1977).

20. G.K. Batchelor and J.T. Green, J. Fluid Mech., 56(3), 401 (1972).

21. D.J. Jeffrey and A. Acrivos, AICHE J., 22(3), 417 (1976).

22. R.P. Chhabra, "Non-Newtonian fluids: An introduction," in Rheology of complex fluids, Springer, New York (2010).

23. M.M. Denn, AICHE J., 50(10), 2335 (2004).

24. D.V. Boger, Nature, 265(5590), 126 (1977).

25. X. Chateau, G. Ovarlez, and K.L. Trung, J. Rheol., 52(2), 489 (2008).

26. J. Domurath, M. Saphiannikova, G. Ausias, and G. Heinrich, J. Non-Newton. Fluid Mech., 171-172, 8 (2012).

27. J. Domurath, M. Saphiannikova, J. Férec, G. Ausias, and G. Heinrich, J. Non-Newton. Fluid Mech., 221, 95 (Jul. 2015).

28. R. PAL, Chem. Eng. Comm., 89(1), 209 (Mar. 1990).

29. A. Crié, C. Baritaud, R. Valette, and B. Vergnes, Polym. Eng. Sci., 55(9), 2156 (2015).

30. M.A. Lauffer, J. Chem. Edu., 58, 250 (1981).

31. H.M. Smallwood, J. Appl. Phys., 15(11), 758 (1944).

32. "Viscosity and Newton's Law of Viscous Force - Fluid Mechanics and Surface Tension - Cengage Learning - JEE Main 2015 Complete Course," GradeStack Courses. [Online]. Available: http://gradestack.com/JEE-Main-2015-Complete/Fluid-

Mechanics-and/Viscosity-and-Newtons/19498-3784-41146-study-wtw. [Accessed: 21-Sep-2017].

33. "Mise en forme des polymères ( $4^{\circ}$ Éd.) AGASSANT Jean-François, AVENAS Pierre, SERGENT Jean-Philippe, VERGNES Bruno, VINCENT Michel," Librairie Lavoisier. [Online]. Available: https://www.lavoisier.fr/livre/industries-chimiques/mise-enforme-des-polymeres-4-ed/agassant/descriptif-9782743015497. [Accessed: 11-Sep-2017]. 
34. T. Kataoka, T. Kitano, M. Sasahara, and K. Nishijima, Rheol. Acta, 17(2), 149 (1978).

35. A.B. Metzner, J. Rheol., 29(6), 739 (1985).

36. N.D. Polychronopoulos, Z. Charlton, D. Suwanda, and J. Vlachopoulos, Adv. Polym. Technol., 37(4), 1161 (2018).

37. R. Riesen, "Détermination de la capacité calorifique aux températures élevées à l'aide de la TGA/DSC Partie 1: méthode DSC standard." Available at: www.mt.com/ta

38. M. C. Le, S. Belhabib, C. Nicolazo, P. Vachot, and A. Sarda, "Identification de la conductivité thermique d'un polymère semi- cristallin en exploitant la mesure de température au c ur d'une pièce moulée par injection," 20ème Congrès Fr. Mécanique 28 Août2 Sept 2011-25044 Besançon Fr. FR, 2011.

39. E. Bonneau, "Contribution a l'etude des proprietes rheologiques des polymeres sur presse a injecter," thesis, Strasbourg 1, 1998.

40. J. Launay, N. Allanic, P. Mousseau, R. Deterre, and Y. Madec, Polym. Eng. Sci., 54(12), 2806 (2014).

41. C. Han, Multiphase Flow in Polymer Processing, Academic Press, New York (1981).

42. J. Laven and H.N. Stein, J. Rheol., 35, 1523 (1991). 